\title{
Dynamic responses of a layered medium subjected to anti-plane loadings
}

\author{
Chien-Ching $\mathrm{Ma}^{*}$, Shaw-Wen Liu, Genn-Sheng Lee \\ Department of Mechanical Engineering, National Taiwan University, 1 Roosevelt Road, Sec 4, Taipei 10617, Taiwan, ROC
}

Received 22 May 2000; in revised form 29 March 2001

\begin{abstract}
The dynamic response of a layered medium subjected to anti-plane loadings is investigated in this study. An effective analytical method is used to present transient full field solutions in the layered medium. The boundary value problem is solved using Laplace transform with respect to time and Fourier transform with respect to space. The transient response is then implemented analytically by Cagniard's method that obviates the contour integration difficulties and whose terms represent successively reflected and transmitted waves. The static solution is obtained by the extreme case of transient response while the time is sufficiently long. The objective in this study is to investigate the transition phenomena from transient response to static value. The numerical examples of a layered half-space are illustrated and discussed in detail. It is found that the characteristic time of the transition not only depends on the distance between source and receiver but also is strongly related to the wave velocities of layer and half-space. Furthermore, very interesting transition behavior is observed when the layer has faster wave velocity than the half-space. () 2001 Elsevier Science Ltd. All rights reserved.
\end{abstract}

Keywords: Layered medium; Transient response; Anti-plane loadings; Full field solutions

\section{Introduction}

The study of wave propagation in multilayered solid materials is of considerable interest in various technological applications. In aerospace and mechanical engineering applications, the analysis of the response of certain structural elements composed of laminated composites subjected to dynamic loads is often carried out on layered plate models. In earth geophysics and earthquake engineering, the analysis and synthesis of the ground motion generated by explosions and earthquakes are usually based on earth models composed of a stack of homogeneous layers overlying on a uniform half-space. The coated layer materials are also of considerable importance in electronic engineering applications. In some practical problems, the static field is obtained after transient effects die away. How long it takes and what the transition behavior is from transient response to static value are significant from the practical point of view.

\footnotetext{
${ }^{*}$ Corresponding author. Tel.: +886-2-2365-9996; fax: +886-2-2363-1755.

E-mail address: cma@w3.me.ntu.edu.tw (C.-C. Ma).
} 
A variety of mathematical tools have been developed to analyze the wave propagation in layered media and to understand the mechanical processes associated with the generation of the elastic waves at the source and their subsequent transformations as they propagate through the layers. The classical analysis in this area was first reported by Lamb (1904), in which the response produced by a transient load on the surface of a uniform half-space was studied by means of an integral transform technique followed by the analytical evaluation of the inversion integrals. Since then, analytical treatments of more complex problems involving one or more layers continued throughout the first half of the 20th century. An excellent description of these early studies can be found in Ewing et al. (1957) and Brekhovskikh (1980).

A matrix method for investigating elastic wave propagation in multilayered media was introduced by Thomson (1950) and subsequently corrected and improved by Haskell (1953). This matrix method was widely used by seismologists to determine the dispersive characteristics of surface waves in layered halfspace models of the earth. Later Gilbert and Backus (1966) introduced the propagator matrix and provided a more formal mathematical background to the technique. Based on the propagator matrix method, a reflection matrix method is proposed by Kennett and Kerry (1979) to construct the entire response in terms of reflection and transmission matrices. This method was then used to analyze the excitation due to general sources in stratified medium. Ma and Huang (1995) derived the transfer relation expressed for the general representations of the response between each layer, instead of displacement-traction vector, to determine the transient response of a layered medium.

The generalized ray theory was developed since 1939 when Cagniard (1939) studied the transient waves in two homogeneous half-spaces in contact. In his monumental work, he had shown that by going through a sequence of contour deformations and changes of integration variables, one is able to find the inverse Laplace transforms of the expressions for each ray. A review of this theory was given by Pao and Gajewski (1977).

Spencer (1960) used the generalized ray method to investigate the surface response of a stratified halfspace to the radiation from a localized source. The method leads to an infinite series of the generalized ray integral constructed in the Laplace transform domain by assembling the source function, reflection and transmission coefficient, the receiver function, and the phase function. The method therefore obviates the necessity for solving a tedious boundary value problem. The time function associated with each ray integral is obtained by using the Cagniard method (Cagniard, 1939). The transient surface response of a layered half-space due to an SH torque pulse from a point source situated inside the layer was obtained by Pekeris et al. (1963). A comprehensive study of transient waves in a multilayered medium based on the generalized ray theory was made by Müller (1968a,b, 1969) for various loading conditions. Recently, the transient responses of a layered medium subjected to in-plane loadings were investigated analytically by Lee and Ma (2000) using a matrix method. The connection between the matrix method and the generalized ray method was established for the layered medium in the transform domain. Then, the numerical and experimental results for a layered half-space were presented by Ma and Lee (2000).

In this study, the transient responses of a multilayered medium subjected to arbitrarily distributed antiplane loadings are investigated by an effective matrix method developed by Lee and Ma (1998). The solution in transform domain is constructed in a form of power series of the phase-related reflection and transmission matrix. These terms represent successively reflected and transmitted waves. The transient response is thus obtained by the application of Cagniard's method. Although the number of waves in the layered medium is infinite, the solution is exact up to the arrival time of the next ray. The static solution is obtained by applying the final value theorem to the transient solution with Heaviside loading in the transform domain. For quantitative analysis, the responses of a layered half-space subjected to an antiplane loading are investigated in detail. Both transient and static responses are presented in closed form solutions. The layered half-space with two different wave velocity ratios between layer and half-space is considered to show the very distinct transition phenomena from transient responses to static values. The 
numerical results are also used to investigate the characteristic time during which the transient effect is important.

\section{Formulation of a layered medium}

Consider an $n$-layered medium as shown in Fig. 1. We assume that the layers are elastic, homogeneous, and isotropic and the interfaces of the layers are perfectly welded. The undisturbed medium is subjected to anti-plane loadings at time $t=0$. The quantities related to $i$ th layer are suffixed by a superscript or subscript (i) while those related to $i$ th interface are attached by a superscript [i]. Since responses for the medium subjected to dynamic loadings located within layers can be obtained by introducing artificial interfaces at the applied-loading locations, we consider first that all applied loadings are located at interfacial or lateral surfaces of the layered medium.

\subsection{Governing equation}

For the anti-plane problem, the governing equation in any layer under no body forces can be expressed as follows:

$$
\frac{\partial^{2} w}{\partial x^{2}}+\frac{\partial^{2} w}{\partial y^{2}}=s_{T}^{2} \frac{\partial^{2} w}{\partial t^{2}}
$$

where $w$ is the out-of-plane displacement and $s_{T}$ is the slowness of the shear wave given by

$$
s_{T}=1 / c_{T}=\sqrt{\rho / \mu},
$$

in which $c_{T}, \rho$, and $\mu$ are the shear wave velocity, mass density, and shear modulus, respectively. The relevant stress components follow the Hooke's law

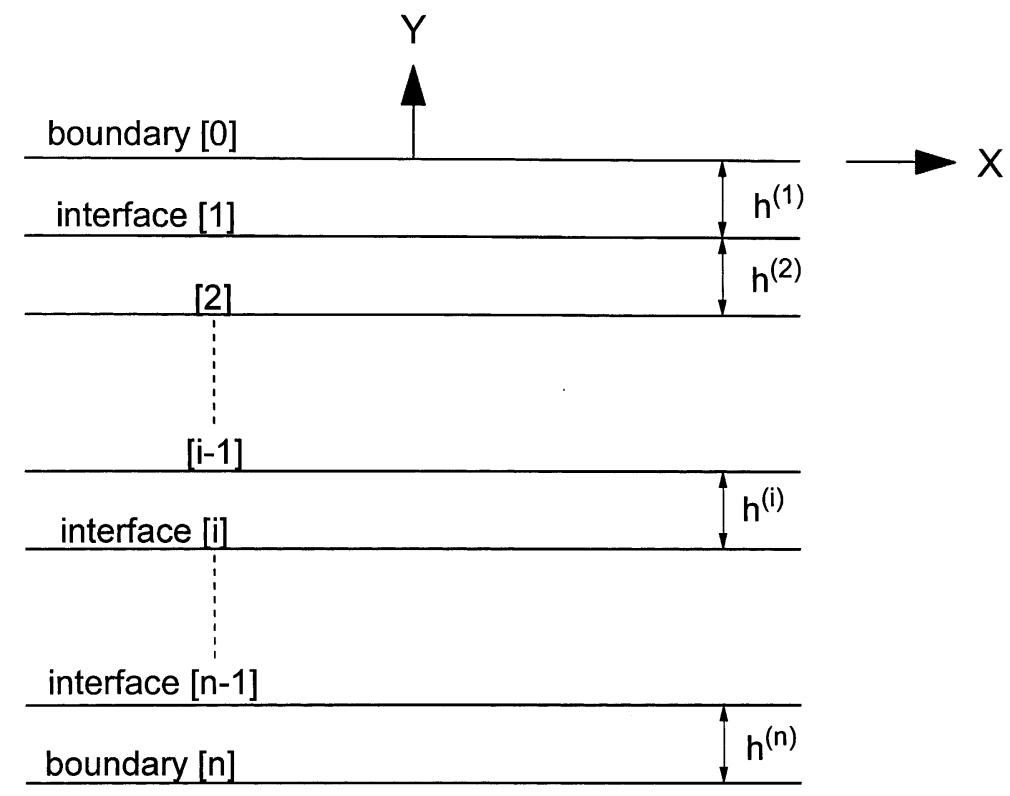

Fig. 1. Configuration and coordinate system of an $n$-layered medium. 


$$
\sigma_{x z}=\mu \frac{\partial w}{\partial x}, \quad \sigma_{y z}=\mu \frac{\partial w}{\partial y} .
$$

\subsection{Boundary conditions}

The boundary conditions on the surfaces of top and bottom layers of the multilayered medium are given by

$$
\begin{aligned}
& \sigma_{y z}^{(1)}(x, 0, t)=\sigma_{y z}^{[0]}(x, t), \quad \text { for }-\infty<x<\infty, \\
& \sigma_{y z}^{(n)}\left(x,-h_{n}, t\right)=\sigma_{y z}^{[n]}(x, t), \quad \text { for }-\infty<x<\infty,
\end{aligned}
$$

where

$$
h_{n}=\sum_{i=1}^{n} h^{(i)}
$$

in which $h^{(i)}$ is the thickness of the $i$ th layer. Loadings applied at the interface $y=-h_{i}$ between two adjacent layers yield the traction and displacement discontinuity conditions,

$$
\begin{aligned}
& w^{(i)}\left(x,-h_{i}, t\right)-w^{(i+1)}\left(x,-h_{i}, t\right)=w^{[i]}(x, t), \quad i=1,2,3, \ldots, n-1, \\
& \sigma_{y z}^{(i)}\left(x,-h_{i}, t\right)-\sigma_{y z}^{(i+1)}\left(x,-h_{i}, t\right)=\sigma_{y z}^{[i]}(x, t), \quad i=1,2,3, \ldots, n-1 .
\end{aligned}
$$

The applied displacement-traction vector $\mathbf{t}^{[i]}(i=0,1, \ldots, n)$ at the $n+1$ planes are introduced to simplify our expression,

$$
t^{[0]}=\sigma_{y z}^{[0]}, \quad t^{[n]}=\sigma_{y z}^{[n]},
$$

and

$$
\mathbf{t}^{[i]}=\left(w^{[i]} \sigma_{y z}^{[i]}\right)^{\mathrm{T}}, \quad \text { for } i=1,2, \ldots, n-1 .
$$

When a dynamic concentrated anti-plane force is applied at the $i$ th interface, the discontinuity is given by

$$
\mathbf{t}^{[i]}=(0-f(t) \delta(x))^{\mathrm{T}} .
$$

\subsection{Formulations}

The boundary value problem described above is solved by applying the Laplace transform over time $t$ with transform parameter $p$ and the Fourier transform over spatial coordinate $x$ with $\xi$. The double transform of an arbitrarily function $f(x, y, t)$ is defined by

$$
\hat{f}(y ; \xi, p)=\int_{-\infty}^{\infty} \mathrm{e}^{\mathrm{i} \xi x} \int_{0}^{\infty} f(x, y, t) \mathrm{e}^{-p t} \mathrm{~d} t \mathrm{~d} x,
$$

where $p$ is a positive real number, large enough to ensure the convergence of the integral, and $\xi$ is a complex variable. By applying the double transform, the governing equation (1) becomes an ordinary differential equation with the following general solution

$$
\hat{w}(y ; \xi, p)=w_{-}(\xi, p) \mathrm{e}^{+\gamma_{T} y}+w_{+}(\xi, p) \mathrm{e}^{-\gamma_{T} y},
$$

where $\gamma_{T}=\left(\left(p s_{T}\right)^{2}+\xi^{2}\right)^{1 / 2}$ and the radical is chosen so that $\operatorname{Re} \gamma_{T} \geqslant 0$. $w_{-}$and $w_{+}$are two field coefficients determined by boundary and interface conditions. 
From Eqs. (2) and (8), we obtain the relations between response functions (such as displacement and stress components) and field coefficients in the transform domain as follows (Lee and Ma, 1998):

$$
\left(\begin{array}{c}
\hat{w}(y) \\
\cdots \hat{\sigma}_{y z}(y)
\end{array}\right)=\left(\begin{array}{c}
M_{11}(y) M_{12}(y) \\
\hdashline M_{21}(y) M_{22}(y)
\end{array}\right)\left(\begin{array}{c}
w_{-} \\
\cdots \\
w_{+}
\end{array}\right)
$$

where

$$
\begin{aligned}
& M_{11}(y ; \xi, p)=\mathrm{e}^{+\gamma_{T} y}, \\
& M_{12}(y ; \xi, p)=\mathrm{e}^{-\gamma_{T} y}, \\
& M_{21}(y ; \xi, p)=\mu \gamma_{T} \mathrm{e}^{+\gamma_{T} y}, \\
& M_{22}(y ; \xi, p)=-\mu \gamma_{T} \mathrm{e}^{-\gamma_{T} y},
\end{aligned}
$$

are phase-related receiver elements.

From boundary and interface conditions in Eqs. (3a), (3b), (4a) and (4b) and applying the double integral transform, a system of equations are developed to determine the field coefficients $w_{-}^{(i)}$ and $w_{+}^{(i)}$ for each layer as follows:

$$
\begin{aligned}
& \left(M_{21}^{(1)}(0) M_{22}^{(1)}(0)\right)\left(\begin{array}{l}
w_{-}^{(1)} \\
w_{+}^{(1)}
\end{array}\right)=\hat{t}^{[0]} \text { at top surface } y=0, \\
& \left(M_{21}^{(n)}\left(-h_{n}\right) M_{22}^{(n)}\left(-h_{n}\right)\right)\left(\begin{array}{l}
w_{-}^{(n)} \\
w_{+}^{(n)}
\end{array}\right)=\hat{t}^{[n]} \text { at bottom surface } y=-h_{n}, \\
& \left(\begin{array}{llll}
M_{11}^{(i)}\left(-h_{i}\right) & M_{12}^{(i)}\left(-h_{i}\right) & -M_{11}^{(i+1)}\left(-h_{i}\right) & -M_{12}^{(i+1)}\left(-h_{i}\right) \\
M_{21}^{(i)}\left(-h_{i}\right) & M_{22}^{(i)}\left(-h_{i}\right) & -M_{21}^{(i+1)}\left(-h_{i}\right) & -M_{22}^{(i+1)}\left(-h_{i}\right)
\end{array}\right)\left(\begin{array}{l}
w_{-}^{(i)} \\
w_{+}^{(i)} \\
w_{-}^{(i+1)} \\
w_{+}^{(i+1)}
\end{array}\right)=\hat{\mathbf{t}}^{[i]} \quad \text { at interface } y=-h_{i}, \\
& i=1,2, \ldots, n-1,
\end{aligned}
$$

where $\hat{\mathbf{t}}^{[i]}$ indicates the transformed field for applied displacement-traction vector $\mathbf{t}^{[i]}$ as defined in Eq. (5). Introducing a global field vector $\mathbf{c}(2 n$ elements) for the multilayered medium,

$$
\mathbf{c}(\xi, p)=\left(\left(\begin{array}{l}
w_{-}^{(1)}(\xi, p) \\
w_{+}^{(1)}(\xi, p)
\end{array}\right)^{\mathrm{T}} \cdots\left(\begin{array}{l}
w_{-(n)}^{(n)}(\xi, p) \\
w_{+}^{(n)}(\xi, p)
\end{array}\right)^{\mathrm{T}}\right)^{\mathrm{T}},
$$

and the global boundary displacement-traction vector $\hat{\mathbf{t}}$,

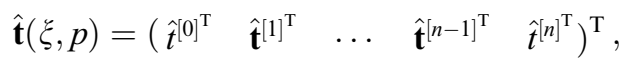

then Eq. (11) can be rewritten in a more compact form

$$
\mathbf{M c}=\hat{\mathbf{t}},
$$

where the coefficient matrix $\mathbf{M}$ is a $2 n \times 2 n$ matrix given by (Lee and Ma, 1998) 


$$
\mathbf{M}=\mathbf{D}+\mathbf{L}+\mathbf{U}=\left[\begin{array}{lllllll}
\mathbf{D}_{0} & \mathbf{U}_{0} & & & & & \\
\mathbf{L}_{1} & \mathbf{D}_{1} & \mathbf{U}_{1} & & & & \\
& \mathbf{L}_{2} & \mathbf{D}_{2} & \ddots & & & \\
& & \ddots & \ddots & \ddots & & \\
& & & \ddots & \mathbf{D}_{n-2} & \mathbf{U}_{n-2} & \\
& & & & \mathbf{L}_{n-1} & \mathbf{D}_{n-1} & \mathbf{U}_{n-1} \\
& & & & & \mathbf{L}_{n} & \mathbf{D}_{n}
\end{array}\right]
$$

In Eq. (15), the components of diagonal matrix $\mathbf{D}$ are given by

$$
\begin{aligned}
\mathbf{D}_{0} & =M_{21}^{(1)}(0) \\
\mathbf{D}_{i} & =\left[\begin{array}{ll}
M_{12}^{(i)}\left(-h_{i}\right) & -M_{11}^{(i+1)}\left(-h_{i}\right) \\
M_{22}^{(i)}\left(-h_{i}\right) & -M_{21}^{(i+1)}\left(-h_{i}\right)
\end{array}\right] \quad(i=1,2, \ldots, n-1), \\
\mathbf{D}_{n} & =M_{22}^{(n)}\left(-h_{n}\right),
\end{aligned}
$$

the nonzero block elements of upper triangular matrix $\mathbf{U}$ are

$$
\begin{aligned}
\mathbf{U}_{0} & =\left[\begin{array}{ll}
M_{22}^{(1)}(0) & 0
\end{array}\right], \\
\mathbf{U}_{i} & =\left[\begin{array}{ll}
-M_{12}^{(i+1)}\left(-h_{i}\right) & 0 \\
-M_{22}^{(i+1)}\left(-h_{i}\right) & 0
\end{array}\right] \quad(i=1,2, \ldots, n-2), \\
\mathbf{U}_{n-1} & =\left[\begin{array}{l}
-M_{12}^{(n)}\left(-h_{n-1}\right) \\
-M_{22}^{(n)}\left(-h_{n-1}\right)
\end{array}\right],
\end{aligned}
$$

and the nonzero blocks for lower triangular matrix $\mathbf{L}$ are defined as

$$
\begin{aligned}
\mathbf{L}_{1} & =\left[\begin{array}{l}
M_{11}^{(1)}\left(-h_{1}\right) \\
M_{21}^{(1)}\left(-h_{1}\right)
\end{array}\right], \\
\mathbf{L}_{i} & =\left[\begin{array}{ll}
0 & M_{11}^{(i)}\left(-h_{i}\right) \\
0 & M_{21}^{(i)}\left(-h_{i}\right)
\end{array}\right] \quad(i=2,3, \ldots, n-1), \\
\mathbf{L}_{n} & =\left[\begin{array}{ll}
0 & M_{21}^{(n)}\left(-h_{n}\right)
\end{array}\right] .
\end{aligned}
$$

Note that the diagonal block matrix $\mathbf{D}$ is a nonsingular matrix. The stacked matrix equation as indicated in Eq. (14) can be solved directly by.

$$
\mathbf{c}=\mathbf{M}^{-1} \hat{\mathbf{t}} \text {. }
$$

Once the global field vector $\mathbf{c}$ is obtained, the response functions in each layer can be determined immediately.

The coefficient matrix $\mathbf{M}$ can be written in an alternative form by extracting the diagonal block matrix $\mathbf{D}$ out of the expression as (Lee and Ma, 1998) 


$$
\mathbf{M}=\mathbf{D}(\mathbf{I}-\mathbf{R}),
$$

where the matrix $\mathbf{R}$ is given by,

$$
\mathbf{R}=-\mathbf{D}^{-1}(\mathbf{L}+\mathbf{U})
$$

or alternatively,

$$
\mathbf{R}=\left[\begin{array}{ccccccc}
0 & -\mathbf{D}_{0}^{-1} \mathbf{U}_{0} & & & & & \\
-\mathbf{D}_{1}^{-1} \mathbf{L}_{1} & \mathbf{0}_{2 \times 2} & -\mathbf{D}_{1}^{-1} \mathbf{U}_{1} & & & & \\
& -\mathbf{D}_{2}^{-1} \mathbf{L}_{2} & \mathbf{0}_{2 \times 2} & \ddots & & & \\
& & \ddots & \ddots & \ddots & & \\
& & & \ddots & \mathbf{0}_{2 \times 2} & -\mathbf{D}_{n-2}^{-1} \mathbf{U}_{n-2} & \\
& & & & -\mathbf{D}_{n-1}^{-1} \mathbf{L}_{n-1} & \mathbf{0}_{2 \times 2} & -\mathbf{D}_{n-1}^{-1} \mathbf{U}_{n-1} \\
& & & & & -\mathbf{D}_{n 1}^{-1} \mathbf{L}_{n} & 0
\end{array}\right] .
$$

The global field vector $\mathbf{c}$ is then expressed by

$$
\mathbf{c}=(\mathbf{I}-\mathbf{R})^{-1} \mathbf{s},
$$

where the source vector $\mathbf{s}$ is given by

$$
\mathbf{s}=\mathbf{D}^{-1} \hat{\mathbf{t}}
$$

or, in a component form,

$$
\mathbf{s}(\xi, p)=\left(\begin{array}{c}
\mathbf{D}_{0}^{-1} \hat{\mathbf{t}}^{[0]} \\
\mathbf{D}_{1}^{-1} \hat{\mathbf{t}}^{[1]} \\
\vdots \\
\mathbf{D}_{n-1}^{-1} \hat{\mathbf{t}}^{[n-1]} \\
\mathbf{D}_{n}^{-1} \hat{\mathbf{t}}^{[n]}
\end{array}\right) .
$$

By the expansion of the inversion matrix of $(\mathbf{I}-\mathbf{R})$ in Eq. (23) into power matrix series of $\mathbf{R}$, we obtain (Lee and Ma, 1998)

$$
\mathbf{c}=\sum_{i=0}^{\infty} \mathbf{R}^{i} \mathbf{s}
$$

It can be shown that the elements of $\mathbf{R}$ shown in Eq. (22) are related to the phase-related reflection and transmission coefficients. First, the general waves propagating toward the interface $[i]$ from the upper medium $(i)$ are considered. By the application of the continuity conditions at the interface, the phase-related reflection coefficient $R_{i / i+1}$ at the interface [i] is expressed as follows:

$$
R_{i / i+1}(\xi, p)=r_{i / i+1} \mathrm{e}^{-2 \gamma_{T(i)} h_{i}}, \quad r_{i / i+1}=\frac{\mu_{(i)} \gamma_{T(i)}-\mu_{(i+1)} \gamma_{T(i+1)}}{\mu_{(i)} \gamma_{T(i)}+\mu_{(i+1)} \gamma_{T(i+1)}},
$$

the phase-related transmission coefficient $T_{i / i+1}$ is

$$
T_{i / i+1}(\xi, p)=t_{i / i+1} \mathrm{e}^{-\left(\gamma_{T(i)}-\gamma_{T(i+1)}\right) h_{i}}, \quad t_{i / i+1}=\frac{2 \mu_{(i)} \gamma_{T(i)}}{\mu_{(i+1)} \gamma_{T(i+1)}+\mu_{(i)} \gamma_{T(i)}} .
$$

Note that $r_{i / i+1}$ and $t_{i / i+1}$ are the reflection and transmission coefficients for plane waves, respectively. Secondly, consider the incident waves traveling upward to the interface $[i]$ from the lower medium $(i+1)$. Similarly, the phase-related reflected coefficient $R_{i+1 / i}$ is 


$$
R_{i+1 / i}(\xi, p)=r_{i+1 / i} \mathrm{e}^{2 \gamma_{T(i+1)} h_{i}}, \quad r_{i+1 / i}=\frac{\mu_{(i+1)} \gamma_{T(i+1)}-\mu_{(i)} \gamma_{T(i)}}{\mu_{(i+1)} \gamma_{T(i+1)}+\mu_{(i)} \gamma_{T(i)}}
$$

the phase-related transmission coefficient $T_{i+1 / i}$ is

$$
T_{i+1 / i}(\xi, p)=t_{i+1 / i} \mathrm{e}^{\left(\gamma_{T(i)}-\gamma_{T(i+1)}\right) h_{i}}, \quad t_{i+1 / i}=\frac{2 \mu_{(i+1)} \gamma_{T(i+1)}}{\mu_{(i)} \gamma_{T(i)}+\mu_{(i+1)} \gamma_{T(i+1)}} .
$$

The global phase-related reflection and transmission matrix $\mathbf{R}$ given in Eq. (22) can be rewritten in terms of the local reflection and transmission coefficients as follows:

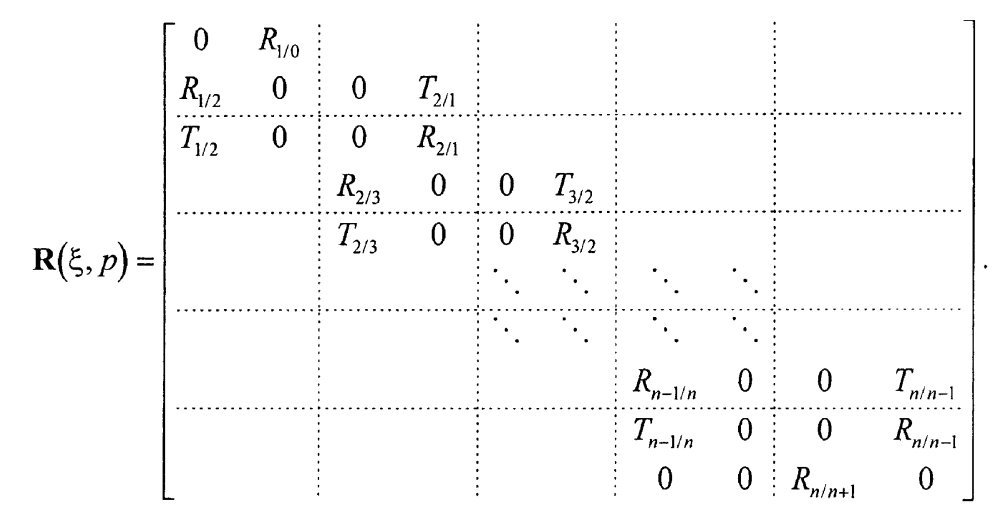

By arranging the response functions in each layer into a response vector, we can relate this vector to the global field vector with a phase-related receiver matrix $\mathbf{R}_{\mathrm{cv}}$. For example, if the response functions $\hat{w}$ and $\hat{\sigma}_{y z}$ in each layer are concerned, we can define the response vector as

$$
\left.\mathbf{b}(y ; \xi, p)=\left(\begin{array}{c}
\hat{w}^{(1)}(y ; \xi, p) \\
\hat{\sigma}_{y z}^{(1)}(y ; \xi, p)
\end{array}\right)^{\mathrm{T}} \quad\left(\begin{array}{c}
\hat{w}^{(2)}(y ; \xi, p) \\
\hat{\sigma}_{y z}^{(2)}(y ; \xi, p)
\end{array}\right)^{\mathrm{T}} \quad \cdots \quad\left(\begin{array}{c}
\hat{w}^{(n)}(y ; \xi, p) \\
\hat{\sigma}_{y z}^{(n)}(y ; \xi, p)
\end{array}\right)^{\mathrm{T}}\right)^{\mathrm{T}}
$$

Thus the response vector can be expressed as

$$
\mathbf{b}(y)=\mathbf{R}_{\mathrm{cv}}(y) \sum_{i=0}^{\infty} \mathbf{R}^{i} \mathbf{s},
$$

where the phase-related receiver matrix is given by

$$
\mathbf{R}_{\mathrm{cv}}(y ; \xi, p)=\left[\begin{array}{cc:c:c:c}
M_{11}^{(1)}(y) & M_{12}^{(1)}(y) & \vdots & \\
M_{21}^{(1)}(y) & M_{22}^{(1)}(y) & & & \\
\hdashline & & M_{11}^{(2)}(y) & M_{12}^{(2)}(y) & \\
& & M_{21}^{(2)}(y) & M_{22}^{(2)}(y) & \\
& & & \ddots & \\
\hdashline & & & M_{11}^{(n)}(y) & M_{12}^{(n)}(y) \\
& & & M_{21}^{(n)}(y) & M_{22}^{(n)}(y)
\end{array}\right]
$$

The solution shown in Eq. (33) is applicable to practical problem since only finite terms out of infinite sum are involved for any given time of interest. 
Next, we consider the response of the layered medium subjected to dynamic loadings that are located within layers instead of at the interface. The solution can be deduced easily by modifying the source vector $\mathbf{s}$ presented in Eq. (25) through considering the physical meaning of the reflection and transmission matrix $\mathbf{R}$ in Eq. (31). Since the representation of the source waves in the infinite domain depends on the location of receiver, the source term in the matrix solution should be separated from the summation and will be denoted by a vector $\mathbf{s}_{0}^{*}$. The source emits waves propagating in two directions, which will become the incident waves in the successive reflection and transmission by the interfaces. The succeeding reflected or transmitted waves are thus obtained by multiplying the matrix $\mathbf{R}$ with $\mathbf{s}^{*}$. The transformed solution for a body source located at $y=-h_{s_{i}}$ in $i$ th layer is expressed as follows (Lee and Ma, 1998):

$$
\mathbf{b}(y)=\mathbf{R}_{\mathrm{cv}}(y) \mathbf{s}_{0}^{*}+\mathbf{R}_{\mathrm{cv}}(y) \sum_{i=1}^{\infty} \mathbf{R}^{i} \mathbf{s}^{*},
$$

where $\mathbf{s}_{0}^{*}$ is defined as

$$
\begin{aligned}
& \mathbf{s}_{0}^{*}(\xi, p)=\left(0,0, \ldots, 0, \mathbf{s}_{+h_{s_{i}}}^{(i)}, \ldots, 0\right)^{\mathrm{T}} \text { for }-h_{i}>y>-h_{s_{i}}>-h_{i+1} ; \\
& \mathbf{s}_{0}^{*}(\xi, p)=\left(0,0, \ldots, \mathbf{s}_{-h_{s_{i}}}^{(i)}, 0, \ldots, 0,0\right)^{\mathrm{T}} \text { for }-h_{i}>-h_{s_{i}}>y>-h_{i+1} ;
\end{aligned}
$$

and $\mathbf{s}^{*}$ is given by

$$
\mathbf{s}^{*}(\xi, p)=\left(0,0, \ldots, \mathbf{s}_{-h_{s_{i}}}^{(i)}, \mathbf{s}_{+h_{s_{i}}}^{(i)}, \ldots, 0,0\right)^{\mathrm{T}} \text { for all } y .
$$

The elements of source vector for an anti-plane concentrated force with unit step time dependence are given by

$$
\mathbf{s}_{-h_{s_{i}}}^{(i)}=\frac{1}{2 p \mu_{(i)} \gamma_{T(i)}} \mathrm{e}^{\gamma_{T(i)} h_{s_{i}}}, \quad \mathbf{s}_{+h_{s_{i}}}^{(i)}=\frac{1}{2 p \mu_{(i)} \gamma_{T(i)}} \mathrm{e}^{-\gamma_{T(i)} h_{s_{i}}} .
$$

The transient solution can be accomplished by the application of Cagniard's method which is surveyed in detail in the text book by Achenbach (1980). If the applied loadings stated in the corresponding dynamic problem is a unit step time function, the static solution can be deduced from the dynamic solution by the final value theorem for Laplace transform,

$$
\bar{w}^{(i)}(y ; \xi)=\lim _{p \rightarrow 0} p \hat{w}^{(i)}(y ; \xi, p) .
$$

\section{Transient and static solutions for a layered half-space}

In this section, the transient and static solutions of a layer overlying a half-space subjected to an antiplane loading are given explicitly. The transient responses are considered first. At time $t=0$, a concentrated anti-plane force with magnitude $\sigma_{0}$ is applied suddenly at $(x, y)=\left(0,-h_{\mathrm{s}}\right)$ within the top layer. The thickness of the top layer is $h$ and the time dependence of the loading is represented by the Heaviside step time function $H(t)$. The global field vector is a three-elements vector, $\mathbf{c}=\left(w_{-}^{(1)} w_{+}^{(1)} w_{-}^{(2)}\right)^{\mathbf{T}}$, and the source vector is given by

$$
\mathbf{s}_{0}^{*}=\left(\frac{\sigma_{0}}{2 p \mu_{(1)} \gamma_{T(1)}} \mathrm{e}^{+\gamma_{T(1)} h_{\mathrm{s}}} \quad 0 \quad 0 \quad\right)^{\mathrm{T}} \text { for } 0>-h_{\mathrm{s}}>y>-h
$$




$$
\mathbf{s}_{0}^{*}=\left(\begin{array}{lll}
0 & \frac{\sigma_{0}}{2 p \mu_{(1)} \gamma_{T(1)}} \\
\mathrm{e}^{-\gamma_{T(1)} h_{\mathrm{s}}} & 0
\end{array}\right)^{\mathrm{T}} \quad \text { for } 0>y>-h_{\mathrm{s}}>-h,
$$

and

$$
\mathbf{s}^{*}=\left(\frac{\sigma_{0}}{2 p \mu_{(1)} \gamma_{T(1)}} \mathrm{e}^{+\gamma_{T(1)} h_{\mathrm{s}}} \frac{\sigma_{0}}{2 p \mu_{(1)} \gamma_{T(1)}} \mathrm{e}^{-\gamma_{T(1)} h_{\mathrm{s}}} \quad 0\right)^{\mathrm{T}} \quad \text { for all } y .
$$

The phase-related reflection and transmission matrix $\mathbf{R}$, which is independent of the loading conditions, is represented by a $3 \times 3$ matrix as follows,

$$
\mathbf{R}(\xi, p)=\left[\begin{array}{ccc}
0 & r_{1 / 0} & 0 \\
r_{1 / 2} \mathrm{e}^{-2 \gamma_{T(1)} h} & 0 & 0 \\
t_{1 / 2} \mathrm{e}^{-\left(\gamma_{T(1)}-\gamma_{T(2)}\right) h} & 0 & 0
\end{array}\right],
$$

where the reflection coefficient $r_{1 / 0}$ at free surface $y=0$ is unity and $r_{1 / 2}$ and $t_{1 / 2}$ are given in Eqs. (27) and (28), respectively.

If we only concern the responses in the layer, the response vector is thus chosen as,

$$
\mathbf{b}(y)=\left(\begin{array}{ll}
\hat{w}^{(1)} & \hat{\sigma}_{y z}^{(1)}
\end{array}\right)^{\mathrm{T}},
$$

and the receiver matrix is a $2 \times 3$ matrix expressed as follows:

$$
\mathbf{R}_{\mathrm{cv}}(y)=\left(\begin{array}{ccc}
\mathrm{e}^{\gamma_{T(1)} y} & \mathrm{e}^{-\gamma_{T(1)} y} & 0 \\
\mu_{(1)} \gamma_{T(1)} \mathrm{e}^{\gamma_{T(1)} y} & -\mu_{(1)} \gamma_{T(1)} \mathrm{e}^{-\gamma_{T(1)} y} & 0
\end{array}\right)
$$

By sorting the matrix solution in Eq. (35) into a series form and using the Cagniard method, the transient solutions in the layer are expressed explicitly as follows:

$$
\begin{aligned}
& w^{(1)}(x, y, t)=\frac{\sigma_{0}}{2 \pi \mu_{(1)}} \int_{0}^{t}\left\{\operatorname{Im}\left[\frac{1}{\sqrt{s_{T(1)}^{2}-\eta_{0}^{2}}} \frac{\partial \eta_{0}}{\partial \tau}\right] H\left(\tau-t_{0}\right)+\sum_{j=0}^{\infty} \operatorname{Im}\left[r_{1 / 2}^{j} \frac{1}{\sqrt{s_{T(1)}^{2}-\eta_{j 1}^{2}}} \frac{\partial \eta_{j 1}}{\partial \tau}\right] H\left(\tau-t_{j 1}\right)\right. \\
& +\sum_{j=0}^{\infty} \operatorname{Im}\left[r_{1 / 2}^{j+1} \frac{1}{\sqrt{s_{T(1)}^{2}-\eta_{j 2}^{2}}} \frac{\partial \eta_{j 2}}{\partial \tau}\right] H\left(\tau-t_{j 2}\right)+\sum_{j=0}^{\infty} \operatorname{Im}\left[r_{1 / 2}^{j+1} \frac{1}{\sqrt{s_{T(1)}^{2}-\eta_{j 3}^{2}}} \frac{\partial \eta_{j 3}}{\partial \tau}\right] H\left(\tau-t_{j 3}\right) \\
& \left.+\sum_{j=0}^{\infty} \operatorname{Im}\left[r_{1 / 2}^{j+1} \frac{1}{\sqrt{s_{T(1)}^{2}-\eta_{j 4}^{2}}} \frac{\partial \eta_{j 4}}{\partial \tau}\right] H\left(\tau-t_{j 4}\right)\right\} \mathrm{d} \tau \\
& \sigma_{y z}^{(1)}(x, y, t)=\frac{\sigma_{0}}{2 \pi}\left\{( \pm) \operatorname{Im}\left[\frac{\partial \eta_{0}}{\partial t}\right] H\left(t-t_{0}\right)+\sum_{j=0}^{\infty} \operatorname{Im}\left[r_{1 / 2}^{j} \frac{\partial \eta_{j 1}}{\partial t}\right] H\left(t-t_{j 1}\right)-\sum_{j=0}^{\infty} \operatorname{Im}\left[r_{1 / 2}^{j+1} \frac{\partial \eta_{j 2}}{\partial t}\right] H\left(t-t_{j 2}\right)\right. \\
& \left.-\sum_{j=0}^{\infty} \operatorname{Im}\left[r_{1 / 2}^{j+1} \frac{\partial \eta_{j 3}}{\partial t}\right] H\left(t-t_{j 3}\right)+\sum_{j=0}^{\infty} \operatorname{Im}\left[r_{1 / 2}^{j+1} \frac{\partial \eta_{j 4}}{\partial t}\right] H\left(t-t_{j 4}\right)\right\}
\end{aligned}
$$




$$
\begin{aligned}
\sigma_{x z}^{(1)}(x, y, t)=\frac{\sigma_{0}}{2 \pi} & \left\{\operatorname{Im}\left[\frac{\eta_{0}}{\sqrt{s_{T(1)}^{2}-\eta_{0}^{2}}} \frac{\partial \eta_{0}}{\partial t}\right] H\left(t-t_{0}\right)+\sum_{j=0}^{\infty} \operatorname{Im}\left[r_{1 / 2}^{j} \frac{\eta_{j 1}}{\sqrt{s_{T(1)}^{2}-\eta_{j 1}^{2}}} \frac{\partial \eta_{j 1}}{\partial t}\right] H\left(t-t_{j 1}\right)\right. \\
& +\sum_{j=0}^{\infty} \operatorname{Im}\left[r_{1 / 2}^{j+1} \frac{\eta_{j 2}}{\sqrt{s_{T(1)}^{2}-\eta_{j 2}^{2}}} \frac{\partial \eta_{j 2}}{\partial t}\right] H\left(t-t_{j 2}\right)+\sum_{j=0}^{\infty} \operatorname{Im}\left[r_{1 / 2}^{j+1} \frac{\eta_{j 3}}{\sqrt{s_{T(1)}^{2}-\eta_{j 3}^{2}}} \frac{\partial \eta_{j 3}}{\partial t}\right] H\left(t-t_{j 3}\right) \\
& \left.+\sum_{j=0}^{\infty} \operatorname{Im}\left[r_{1 / 2}^{j+1} \frac{\eta_{j 4}}{\sqrt{s_{T(1)}^{2}-\eta_{j 4}^{2}}} \frac{\partial \eta_{j 4}}{\partial t}\right] H\left(t-t_{j 4}\right)\right\},
\end{aligned}
$$

where $\eta_{0}$ and $\eta_{j 1}, \ldots, \eta_{j 4}$ are functions of time and spatial coordinates and satisfy the following equations,

$$
\sqrt{s_{T(1)}^{2}-\eta_{0}^{2}}\left|y+h_{\mathrm{s}}\right|+\eta_{0} x=t
$$

and

$$
\sqrt{s_{T(1)}^{2}-\eta_{j k}^{2}} y_{j k}+\eta_{j k} x=t \quad(k=1,2,3,4),
$$

respectively. $t_{0}$ and $t_{j k}$ are the arrival times of these waves and $y_{j k}$ is given by

$$
\begin{aligned}
& y_{j 1}=2 j h-\left(y-h_{\mathrm{s}}\right), \\
& y_{j 2}=2(j+1) h+\left(y-h_{\mathrm{s}}\right), \\
& y_{j 3}=2(j+1) h+\left(y+h_{\mathrm{s}}\right),
\end{aligned}
$$

and

$$
y_{j 4}=2(j+1) h-\left(y+h_{\mathrm{s}}\right) .
$$

The correspondent static problem can be obtained by applying the final value theorem to the dynamic solution in the transform domain or by taking the limit analysis $t \rightarrow \infty$ to the solution in time domain. The static solutions, $\sigma_{y z}^{(1)}(x, y)$ and $\sigma_{x z}^{(1)}(x, y)$, are given by

$$
\begin{aligned}
\sigma_{y z}^{(1)}(x, y)=\frac{\sigma_{0}}{2 \pi} & {\left[( \pm) \frac{\left|y+h_{\mathrm{s}}\right|}{x^{2}+\left(y+h_{\mathrm{s}}\right)^{2}}+\sum_{j=0}^{\infty}\left(r_{1 / 2}^{\prime}\right)^{j} \frac{y_{j 1}}{x^{2}+y_{j 1}^{2}}-\sum_{j=0}^{\infty}\left(r_{1 / 2}^{\prime}\right)^{j+1} \frac{y_{j 2}}{x^{2}+y_{j 2}^{2}}\right.} \\
& \left.-\sum_{j=0}^{\infty}\left(r_{1 / 2}^{\prime}\right)^{j+1} \frac{y_{j 3}}{x^{2}+y_{j 3}^{2}}+\sum_{j=0}^{\infty}\left(r_{1 / 2}^{\prime}\right)^{j+1} \frac{y_{j 4}}{x^{2}+y_{j 4}^{2}}\right], \\
\sigma_{x z}^{(1)}(x, y)=\frac{\sigma_{0}}{2 \pi} & {\left[\frac{x}{x^{2}+\left(y+h_{\mathrm{s}}\right)^{2}}+\sum_{j=0}^{\infty}\left(r_{1 / 2}^{\prime}\right)^{j} \frac{x}{x^{2}+y_{j 1}^{2}}+\sum_{j=0}^{\infty}\left(r_{1 / 2}^{\prime}\right)^{j+1} \frac{x}{x^{2}+y_{j 2}^{2}}+\sum_{j=0}^{\infty}\left(r_{1 / 2}^{\prime}\right)^{j+1} \frac{x}{x^{2}+y_{j 3}^{2}}\right.} \\
& \left.+\sum_{j=0}^{\infty}\left(r_{1 / 2}^{\prime}\right)^{j+1} \frac{x}{x^{2}+y_{j 4}^{2}}\right],
\end{aligned}
$$

where $r_{1 / 2}^{\prime}=\left(\mu_{(1)}-\mu_{(2)}\right) /\left(\mu_{(1)}+\mu_{(2)}\right)$ is the 'static' reflection coefficient. Note that the dynamic solutions in Eqs. (43b) and (43c) are converged to the static solutions in Eqs. (45a) and (45b) term by term. 


\section{Numerical results and discussions}

In this section, the responses of a layered half-space subjected to an anti-plane loading with Heaviside function are investigated in detail. The thickness of the layer is $h$ and the loading is applied at $(x, y)=$ $(0,-h / 2)$ in the layer. Two cases with different wave velocity ratios are taken into account. One has faster wave velocity in the layer and the ratio of velocities is $c_{T(2) /} / c_{T(1)}=0.771$. The other with $c_{T(2)} / c_{T(1)}=1.297$ has faster velocity in the half-space. Note that the wave velocities shown in the numerical results are normalized to the velocity in the layer for both cases. In order to have a better understanding of the transition behavior from transient response to static value, the quantitative responses at near field (five times the thickness of the layer from the source), intermediate field (50 times the thickness), and far field (200 times the thickness) are presented in the following numerical results. The source and most of the receivers considered in this paper are located at the same depth $(y=-h / 2)$ in the layer but different horizontal positions. In fact, comprehensive studies for various locations of sources and receivers and ratios of wave velocities have been done in this study. However, only the most representative results are chosen to present in this paper.

\subsection{Layer with faster wave velocity}

The case with faster wave velocity in the layer is considered in this section. The wavefronts of the incident, reflected, and refracted waves in the layered half-space at the instant $t / s_{T(1)} h=10$ are shown in Fig. 2. For the responses at intermediate or far range in the layer, it is expected from Fig. 2 that a great number of reflected waves will arrive almost at the moment the incident wave arrives. The arrival times of incident waves and subsequent reflected waves can be obtained from Eqs. (44a) and (44b), respectively. Fig. 3 shows the transient responses of the displacements at three different receivers $(x=5 h, 50 h$, and $200 h)$. It is observed obviously that the displacements grow steadily as time increases. Moreover, at the same instant, the displacements at the receivers closer to the source are larger than those farther to the source. Due to the existence of rigid body motion, there are no corresponding static displacements in the problem considered in this study. However, a particularly interesting phenomenon is exhibited in Fig. 3. The displacements change apparently at around $t=x / c_{T(2)}$ instead of $t=x / c_{T(1)}$ when direct incident wave arrives. For instance, the displacement at $x=200 h$ starts to grow at about $t / s_{T(1)} h \approx 250$ instead of 200 .

The near field response of $\sigma_{y z}^{(1)}$ at $x=5 h$ is shown in Fig. 4. It is found that the transient responses of stresses tend to static value uniformly after the arrival of the first few waves. Because of the Heaviside source time function of the applied loading, the stress fields behave as a square root singularity at the incident and reflected wavefronts. For the intermediate field $(x=50 h)$ and far field $(x=200 h)$, the responses of $\sigma_{y z}^{(1)}$ are shown in Figs. 5 and 6 . These results are quite different from those of near field. As was expected, a large number of reflected waves arrive in a very short time right after the incident wave. For instance, it is found that there are 20 reflected waves arriving at the far field receiver $(x=200 h)$ within first

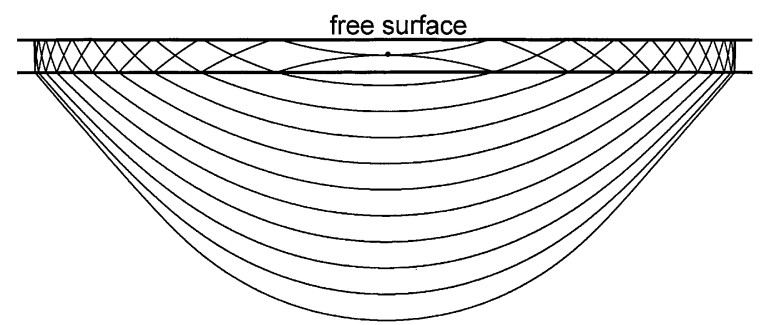

Fig. 2. Wavefronts of the incident, reflected, and refracted waves in a layered half-space $\left(c_{T(2)} / c_{T(1)}=0.771\right)$ at the instant $t / s_{T(1)} h=10$. 


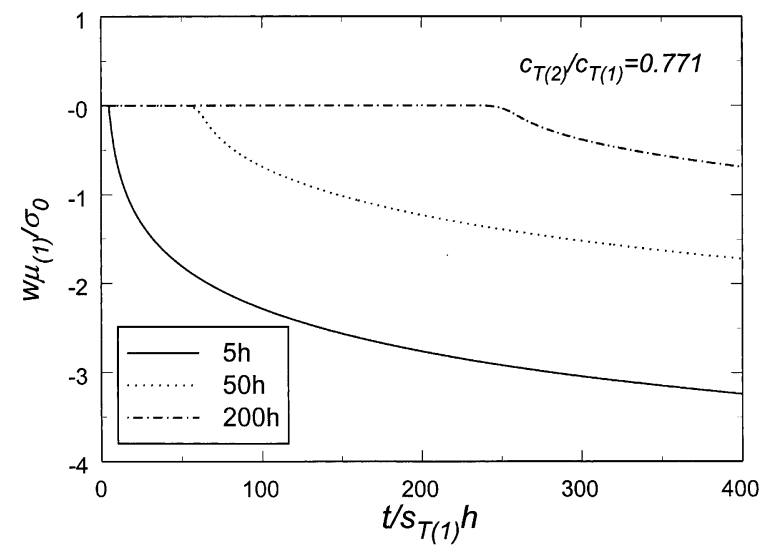

Fig. 3. Transient responses of displacements at $x=5 h, 50 h, 200 h$ and $y=-h / 2$ in the layer with faster wave velocity.

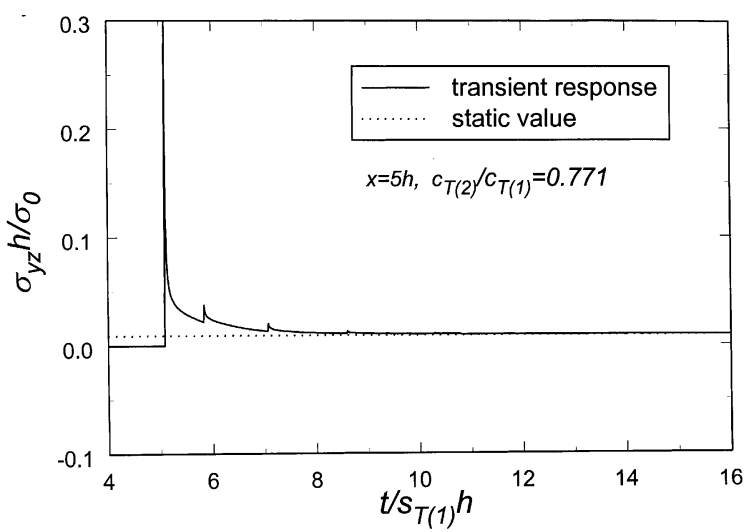

Fig. 4. Transient response and static value of stress $\sigma_{y z}$ at $(x, y)=(5 h,-h / 2)$ in the layer with faster wave velocity.

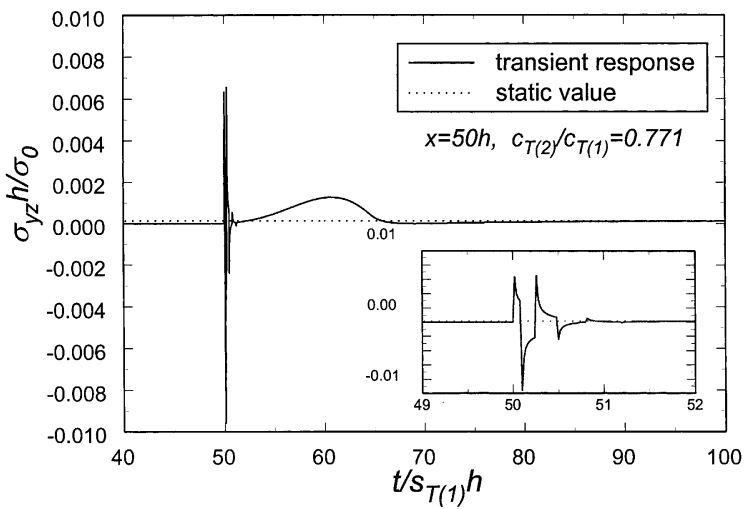

Fig. 5. Transient response and static value of stress $\sigma_{y z}$ at $(x, y)=(50 h,-h / 2)$ in the layer with faster wave velocity. 


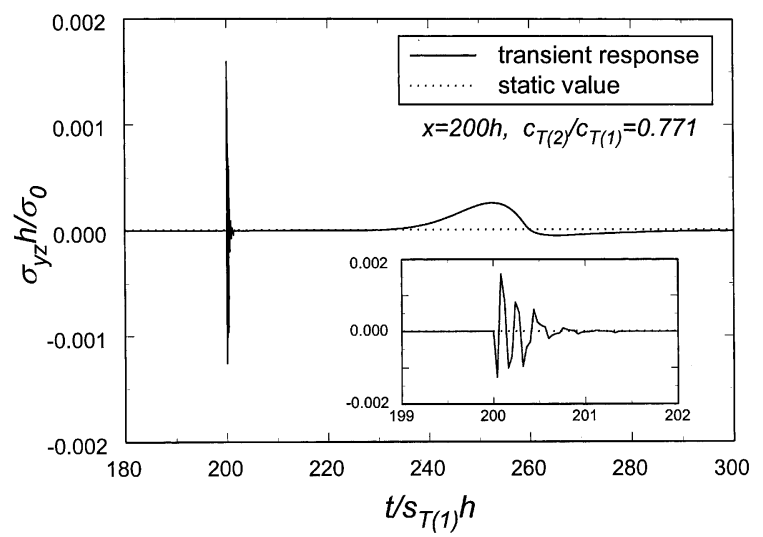

Fig. 6. Transient response and static value of stress $\sigma_{y z}$ at $(x, y)=(200 h,-h / 2)$ in the layer with faster wave velocity.

second (from $t / s_{T(1)} h=200$ to 201). The total number of waves from $t / s_{T(1)} h=200$ to 400 is 693 including one incident wave and 692 reflected waves. This causes the stresses varying tremendously at the very beginning as shown in the small windows in Figs. 5 and 6 . The responses then vary smoothly before going to static value. However, an interesting result is observed in the far field responses shown in Fig. 6. There is a long period $\left(t / s_{T(1)} h \approx 202-230\right)$ while the responses are very small and steady during the transition from transient response to static value. This phenomenon may mislead one to believe that the response has become static value already. In fact, the responses as shown in Fig. 6 will increase gradually after this period and reach a relative maximum value at $t / s_{T(1)} h \approx 252.6$. The relative maximum values is 0.00026 and the corresponding static value is $8.6 \times 10^{-6}$. Finally, the response then goes to static value slowly. It is concluded that the transient responses will go steadily to static value only after $t=x / c_{T(2)}$ (or $t / s_{T(1)} h=$ $\left.x c_{T(1)} / c_{T(2)} h\right)$ which depends not only on the distance $(x)$ between the source and receiver but also on the wave velocity $\left(c_{T(2)}\right)$ in the half-space.

\subsection{Layer with slower wave velocity}

Next, a layered half-space which has a slower wave velocity in the layer is considered. Compared with the last case, the main feature in this case is that the waves in the layer may be refracted along the interface with the higher speed $c_{T(2)}$ and arrive at the receiver earlier than the regular body waves that travel with slower speed $c_{T(1)}$. These waves are well-known head waves. For illustration, the wavefronts of the incident, reflected, refracted, and head waves at $t / s_{T(1)} h=5$ are shown in Fig. 7 where head waves are indicated by dotted lines. Head waves can be observed if the receiver is in a position such that

$$
\frac{x}{R_{j k}}>\frac{c_{T(1)}}{c_{T(2)}}
$$

where $R_{j k}=\left(x^{2}+y_{j k}^{2}\right)^{1 / 2}$ is the distance between receiver and image source. The head wave contributions arise in the evaluation of inverse Laplace transforms along the Cagniard path from $\eta_{j k}=s_{T(2)}$ to $s_{T(1)} x / R_{j k}$. By substituting $\eta_{j k}=s_{T(2)}$ and $s_{T(1)} x / R_{j k}$ into Eq. (44b), the corresponding arrival $\left(t_{j k}^{\mathrm{ha}}\right)$ and end $\left(t_{j k}^{\mathrm{he}}\right)$ times of head waves can be obtained as follows:

$$
\begin{aligned}
& t_{j k}^{\text {ha }}=x s_{T(2)}+y_{j k} \sqrt{s_{T(1)}^{2}-s_{T(2)}^{2}}, \\
& t_{j k}^{\text {he }}=R_{j k} s_{T(1)} .
\end{aligned}
$$




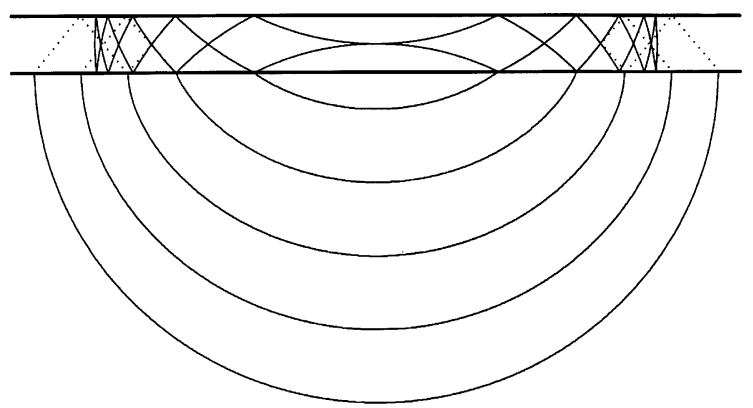

Fig. 7. Wavefronts of the incident, reflected, refracted, and head waves in a layered half-space $\left(c_{T(2)} / c_{T(1)}=1.297\right)$ at the instant $t / s_{T(1)} h=5$.

Note that $t_{j k}^{\text {he }}$ is also the arrival time of the wide-angle reflection. Due to the reflection between free surface and interface, a receiver in the layer may have many head waves propagating through. The arrival time of first head wave $(j=0$ and $k=1)$ then follows from Eq. (47a) as

$$
t_{01}^{\text {ha }}=x s_{T(2)}-\left(y-h_{\mathrm{s}}\right) \sqrt{s_{T(1)}^{2}-s_{T(2)}^{2}} \text {. }
$$

Similarly, from Eqs. (46) and (47b), the end time of the last head wave is given by

$$
t_{\text {last }}^{\text {he }}=\frac{s_{T(1)}^{2}}{s_{T(2)}} x .
$$

The discussions mentioned above will be useful for the understanding of following numerical results.

The transient responses of displacements at three different receivers are shown in Fig. 8. Basically, the responses here have similar pattern to those shown in Fig. 3. The main distinction is the contributions caused by the head waves. The oscillations are clearly observed during the existence of head waves. After the passage of head waves, the displacements increase steadily for the rest of time, in which the reflected body waves exist only.

The transient responses of stress $\sigma_{x z}^{(1)}$ at near $(x=5 h)$, intermediate $(x=50 h)$, and far $(x=200 h)$ fields are shown in Figs. 9-11. These results have totally different characteristics from those of previous case shown in Figs. 4-6. Again, the effect of head waves plays a significant role here. By using Eqs. (48) and (49), we can

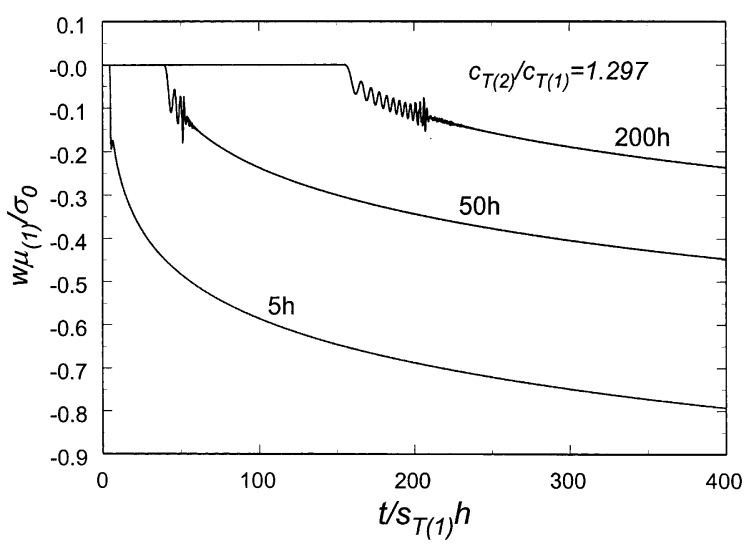

Fig. 8. Transient responses of displacements at $x=5 h, 50 h, 200 h$ and $y=-h / 2$ in the layer with slower wave velocity. 


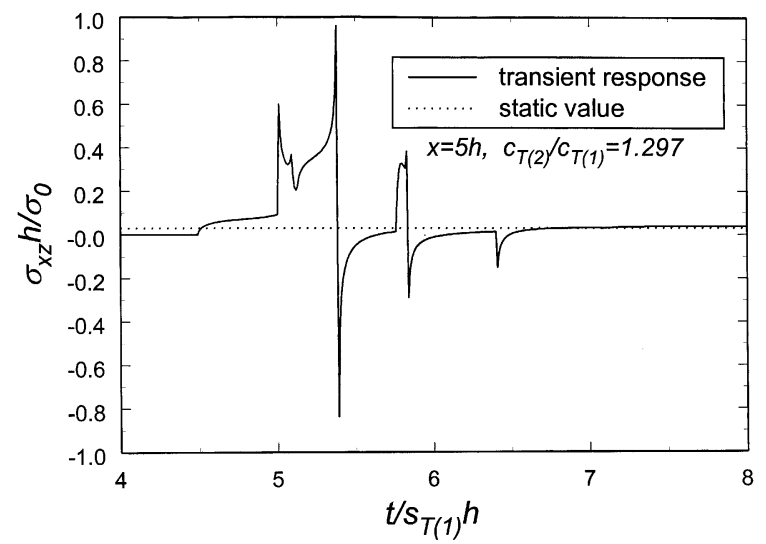

Fig. 9. Transient response and static value of stress $\sigma_{x z}$ at $(x, y)=(5 h,-h / 2)$ in the layer with slower wave velocity.

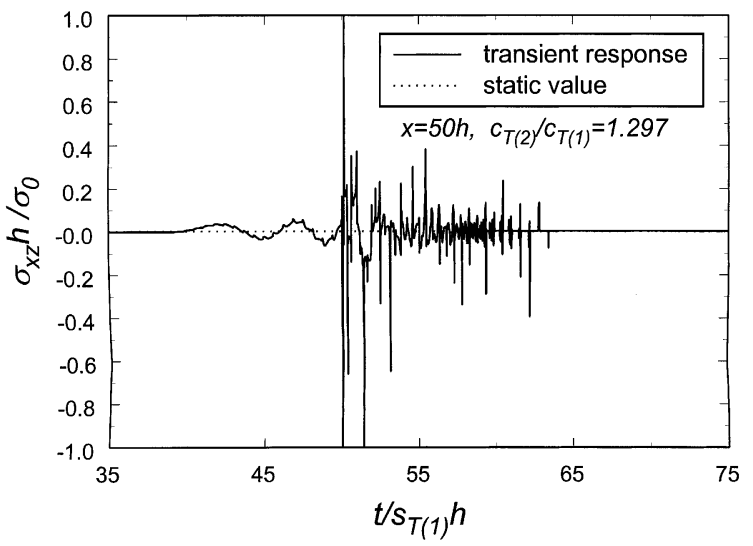

Fig. 10. Transient response and static value of stress $\sigma_{x z}$ at $(x, y)=(50 h,-h / 2)$ in the layer with slower wave velocity.

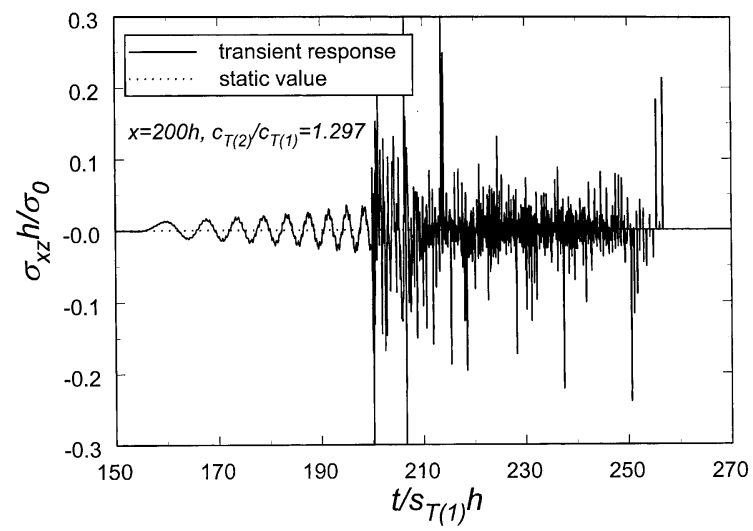

Fig. 11. Transient response and static value of stress $\sigma_{x z}$ at $(x, y)=(200 h,-h / 2)$ in the layer with slower wave velocity. 
estimate that head waves exist from $t / s_{T(1)} h=4.49$ to 6.49 at the receiver $x=5 h$, from 39.19 to 64.85 for $x=50 h$, and from 154.84 to 259.40 for $x=200 h$. It is found that there are 329 head waves arriving at the far-field receiver $(x=200 h)$ from $t / s_{T(1)} h=154.84$ to 259.40 . The characteristic times indicated above can be distinctly observed in the numerical results shown in Figs. 8-11. Most of the transient stresses as shown in Figs. 9-11 oscillate violently during the existence of the singular behavior near the wavefront of body waves and go to static values once the head waves finish, i.e., $t=x c_{T(2)} / c_{T(1)}^{2}$ (or $\left.t / s_{T(1)} h=x c_{T(2)} / c_{T(1)} h\right)$ from Eq. (49). Therefore, in addition to the horizontal distance $(x)$ between source and receiver, the time needed for the transition from transient response to static value in this case depends on both wave velocities in layer and half-space.

\section{Conclusions}

An effective matrix method has been used to investigate the transient waves in a multilayered medium subjected to dynamic anti-plane loadings. The transient responses are obtained by means of Cagnaird's method. In order to understand the physical phenomena in the transition from transient response to static value, the numerical results for two cases of layered half-spaces with different ratios of wave velocities have been investigated in detail. Based on the studies, it is shown that not only the far field but also the long-time responses can be obtained efficiently. It can be concluded from this study that the transient responses go steadily to static values only after $t=x / c_{T(2)}$ for the case with faster wave velocity in the layer and go to static value right after $t=x c_{T(2)} / c_{T(1)}^{2}$ for the case with faster wave velocity in the half-space. A further study for transition from transient response to steady state will be given in a follow-up paper.

\section{Acknowledgements}

The research support of the National Science Council, Republic of China, through Grant NSC 89-2212E002-018 at National Taiwan University is gratefully acknowledged.

\section{References}

Achenbach, J.D., 1980. Wave Propagation in Elastic Solids. North-Holland, Amsterdam.

Brekhovskikh, L.M., 1980. Waves in Layered Media. Academic Press, New York.

Cagniard, L., 1939. Reflexion et Refraction des Ondes Seismiques Progressives. Cauthiers-Villars, Paris (Translated into English and revised by Flinn, E.A., Dix, C.H., 1962. Reflection and Refraction of Progressive Seismic Waves. McGraw Hill, New York).

Ewing, W.M., Jardetzky, W.S., Press, F., 1957. Elastic Waves in Layered Media. McGraw-Hill, New York.

Gilbert, F., Backus, G.E., 1966. Propagator matrices in elastic wave and vibration problems. Geophysics 31, 326-332.

Haskell, N., 1953. The dispersion of surface waves on multilayered media. Bull. Seism. Soc. Am. 43, 17-34.

Kennett, B.L.N., Kerry, N.J., 1979. Seismic waves in a stratified half space. Geophys. J. R. Astron. Soc. 44, $557-583$.

Lamb, H., 1904. On the propagation of tremors over the surface of an elastic solid. Phil. Trans. Roy. Soc. London Ser. A203, 1-42.

Lee, G.S., Ma, C.C., 1998. A new solution methodology for solving dynamic and static antiplane problem of a layered medium. J. CSME 19, 441-455.

Lee, G.S., Ma, C.C., 2000. Transient elastic waves propagating in a multilayered medium subjected to inplane dynamic loadings. Part I: Theory. Proc. R. Soc. London A456, 1355-1374.

Ma, C.C., Huang, K.C., 1995. Wave propagation in layered elastic media for antiplane deformation. Int. J. Solids Struct. 32, $665-678$.

Ma, C.C., Lee, G.S., 2000. Transient elastic waves propagating in a multilayered medium subjected to inplane dynamic loadings. Part II. Numerical calculation and experimental measurement. Proc. R. Soc. London A456, 1375-1396.

Müller, G., 1968a. Theoretical seismograms for some types of point-source in layered media. Part I: Theory. Z. Geophys. 34, 15-35.

Müller, G., 1968b. Theoretical seismograms for some types of point-source in layered media. Part II: Numerical calculations. Z. Geophys. 34, 147-162. 
Müller, G., 1969. Theoretical seismograms for some types of point-source in layered media. Part III: Single force and dipole sources of arbitrary orientation. Z. Geophys. 35, 347-371.

Pao, Y. H., Gajewski, R., 1977. The generalized ray theory and transient responses of layered elastic solids. in: Mason, W.P. (Ed.), Physical Acoustics, vol. 13. Academic Press, New York (Chapter 6).

Pekeris, C.L., Alterman, Z., Abramovici, F., 1963. Propagation of an SH-torque pulse in a layered solid. Bull. Seism. Soc. Am. 53, 3957.

Spencer, T.W., 1960. The method of generalized reflection and transmission coefficients. Geophysics 25, 625-641.

Thomson, W.T., 1950. Transmission of elastic waves through a stratified solid medium. J. Appl. Phys. 21, 89-93. 